\title{
Diagnostica microbiologica delle infezioni associate all'utilizzo di cateteri venosi centrali (CVC): considerazioni generali e studio in pazienti immunocompromessi
}

\author{
Giancarlo Basaglia, Sandro Stocco Calzavara, Antonella Pancino, Paola Sperandio, \\ Maria Luisa Tomasini, Paolo De Paoli
}

Struttura Complessa di Microbiologia, Immunologia e Virologia, Centro di Riferimento Oncologico, Istituto Nazionale Tumori - IRCCS, Aviano (Pordenone)

Microbiological diagnosis of central venous catheter (CVC) infections: general considerations and study in immunocompromised patients

Key words: catheter, infection, diagnosis, immunocompromised host

\section{SUMMARY}

Central venous catheterization today is essential for the intensive management of both medical and surgical patients (for the administration of iv fluids, medications, blood products and parenteral nutrition fluids, to monitor hemodynamic status and to provide hemodialysis). CVC infection is a serious complication of catheterization and a common type of nosocomial infection. This complication is particularly serious in oncologic, haematologic and AIDS, frequently immunocompromised, patients. To prevent CVC infection strategies were proposed, such as use of guidelines for the management of CVC or use of antimicrobialimpregnated CVC. However, despite their high frequency of occurrence and seriousness, CVC infections are often difficult to diagnose. Because of their poor specificity and sensitivity clinical findings are often unreliable for establishing a diagnosis of CVC related infection. For the past 25 years a large number of different microbiologic tests have been proposed. In this paper we report the results of some microbiologic tests (qualitative peripheral and CVC blood culture, qualitative and quantitative CVC tip culture) performed in our Laboratory, in the past 10 years, in oncologic, haematologic and AIDS patients. We report also the results of a study performed by using "quantitative cultures" and "differential time to positive cultures" of peripheral and CVC blood samples. On the basis of findings from our study, quantitative CVC tip culture, "quantitative culture" and "differential time to positive culture" of peripheral and CVC blood samples were found simple, sensitive and specific methods for the diagnosis of CVC and CVC-related infection. The microorganism most commonly associated with these infections is Staphylococcus epidermidis, but also others may be involved such as Pseudomonas aeruginosa, Enterococcus faecalis, and Candida spp.

\section{INTRODUZIONE}

L'utilizzo di cateteri intravascolari è oggi una pratica sempre più diffusa con diverse applicazioni cliniche (terapia con antiblastici, supporto nutrizionale parenterale, monitoraggio emodinamico, irreperibilità di accessi venosi periferici, etc...). Una delle complicanze più importanti di tale pratica sono le infezioni, che rappresentano un importante capitolo nell'ambito delle infezioni correlate alle pratiche assistenziali. Un gruppo particolare di pazienti nei quali si riscontrano tali infezioni sono i pazienti oncologici, ematologici e con AIDS, frequentemente anche immunocompromessi, nei quali la complicanza infettiva associata al cateterismo vascolare può assumere significati di particolare gravità. Per prevenire le infe- zioni dei cateteri vascolari varie sono state le strategie proposte, tra le quali l'elaborazione di linee guida per la gestione dei cateteri e l'utilizzo di cateteri medicati. Nonostante la loro frequenza e gravità $(8,11)$ le infezioni associate al cateterismo vascolare non sono sempre facili da diagnosticare (11). La diagnostica microbiologica si è nel tempo arricchita di tecniche per la diagnosi di tali infezioni alcune utilizzabili prima della rimozione del catetere vascolare (emocoltura qualitativa, emocoltura quantitativa, emocoltura basata sul "differential time to positivity" eseguite su sangue prelevato da catetere e da vena periferica; esame microscopico su sangue prelevato da catetere dopo colorazione di Gram o con arancio di acridina; esami qualitativi o quantitativi di cute o essu- 
dato vicini all'emergenza cutanea del catetere; esami qualitativi o quantitativi dell'hub del catetere) altre dopo la rimozione dello stesso (esame colturale qualitativo, esame colturale semiquantitativo secondo Maki ed esame colturale quantitativo secondo Cleri della punta del catetere). Nel nostro Servizio, al quale afferiscono pazienti quali quelli di un Istituto Nazionale Tumori, abbiamo particolarmente approfondito queste problematiche $(1-4,7,9,10)$.

SCOPI DEL LAVORO: scopi del nostro lavoro sono stati, con riferimento ai pazienti seguiti presso il Centro di Riferimento Oncologico di Aviano (pazienti oncologici, ematologici e con AIDS, frequentemente anche immunocompromessi):

A. Raccolta e riesame dei dati della casistica pluriennale della Struttura Complessa di Microbiologia, Immunologia e Virologia relativi alla diagnostica microbiologica delle infezioni associate al cateterismo vascolare basata sull'emocoltura qualitativa su sangue prelevato da catetere e da vena periferica, sull'esame qualitativo della punta del catetere e, in particolare, sull'esame quantitativo secondo Cleri della punta del catetere;

B. Allestimento ed esecuzione dell'emocoltura quantitativa e dell'emocoltura basata sul "differential time to positivity" eseguite su sangue prelevato da catetere e da vena periferica approfondendone significati e tecniche, pregi e limiti, indicazioni e risultati.

\section{MATERIALI E METODI}

PAZIENTI: i pazienti inclusi in questo studio sono pazienti oncologici con tumori solidi, pazienti oncoematologici e pazienti con AIDS, frequentemente anche immunocompromessi, ricoverati presso il Centro di Riferimento Oncologico (C.R.O.) di Aviano (Pordenone). Ai pazienti sono state eseguite emocolture e l'esame microbiologico della punta del Catetere Venoso Centrale (CVC).

EMOCOLTURE: sono state utilizzate le seguenti tecniche:

- Emocoltura qualitativa;

- Emocoltura quantitativa;

- Emocoltura basata sul "differential time to positivity".

Emocoltura qualitativa

L'emocoltura qualitativa è stata eseguita su sangue prelevato da catetere e da vena periferica utilizzando il Sistema automatico Bactec 9120 (Becton Dickinson) con i flaconi BACTEC PLUS Aerobic/F (per microrganismi aerobi) e i flaconi BACTEC PLUS Anaerobic/F (per microrganismi anaerobi).
Emocoltura quantitativa

L'emocoltura quantitativa è stata eseguita su sangue prelevato da catetere e da vena periferica utilizzando il Sistema Isolator (Oxoid). Sono state utilizzate le provette ISOLATOR ${ }^{\mathrm{TM}} 1.5$ (provette in grado di contenere un volume di sangue pari a $1,5 \mathrm{~mL}$ ). Per ogni paziente sono state inoculate 2 provette ISOLATOR 1.5 con $1,5 \mathrm{~mL}$ di sangue prelevato da CVC e da vena periferica. Per il prelievo da CVC sono stati eliminati i primi $2 \mathrm{~mL}$ di fluido prelevato (che potrebbe contenere farmaci o eparina o soluzione fisiologica che possono compromettere la corretta crescita e conta microbica). I campioni sono stati fatti pervenire al laboratorio entro 15 minuti dal prelievo e processati subito; se questo non è stato possibile sono stati conservati a $+4^{\circ} \mathrm{C}$ per alcune ore onde evitare l'alterazione della carica microbica. Si è proceduto quindi come segue:

a) Sangue periferico:

- semina su Agar Sangue e su Agar

Cioccolato di $0,5 \mathrm{~mL}$ di campione prelevato con pipetta pasteur sterile depositandoli lungo il diametro della piastra; striscio quindi su tutta la superficie con ansa.

\section{b) Sangue prelevato da CVC:}

- Semina su Agar Sangue e su Agar Cioccolato di $10 \mu \mathrm{L}$ di campione prelevato con ansa da $10 \mu \mathrm{L}$ depositandoli lungo il diametro della piastra; striscio quindi su tutta la superficie con l'ansa stessa;

- Semina su Agar Sangue e su Agar Cioccolato di $0,5 \mathrm{~mL}$ di campione prelevato con pipetta pasteur sterile depositandoli lungo il diametro della piastra; striscio quindi su tutta la superficie con l'ansa stessa;

- Allestimento di un vetrino con citocentrifuga con $100 \mu \mathrm{L}$ di campione e colorazione con metodica di Gram (o colorazione con Arancio di acridina);

- Incubazione di tutte le piastre a $37^{\circ} \mathrm{C}$ e $5 \%$ $\mathrm{CO}_{2}$ per 24-48 ore. Se dopo tale periodo non è stata rilevata crescita microbica, l'esame è stato considerato negativo.

Come dimostrato in Letteratura è stato seguito il criterio interpretativo che vi è infezione del catetere se, in presenza di positività di entrambe $\mathrm{i}$ campioni, la carica microbica della coltura da CVC è di almeno 5 volte la carica evidenziata dal sangue periferico.

Emocoltura basata sul "differential time to positivity"

L'emocoltura basata sul "differential time to positivity" (DTP) è stata eseguita utilizzando il Sistema automatico Bactec 9120 (Becton Dickinson), procedendo come segue:

- Inoculo di una coppia di flaconi (flaconi 
BACTEC PLUS Aerobic/F per microrganismi aerobi) e flaconi BACTEC PLUS Anaerobic/F (per microrganismi anaerobi) con $6 \mathrm{~mL}$ di sangue periferico ciascuno, e di una coppia di flaconi con $6 \mathrm{~mL}$ di sangue prelevato da CVC. La quantità di sangue con cui vengono inoculati i flaconi deve essere precisa e la consegna al laboratorio deve avvenire entro 1 ora;

- In Laboratorio inserimento dei flaconi nello strumento entro pochi minuti dalla consegna. Nel momento in cui i flaconi sono accettati dal Sistema tramite il computer gestionale e inseriti nell'incubatore, inizia il tempo di monitoraggio (Time to detection).

Come dimostrato in Letteratura è stato seguito il criterio interpretativo che vi è infezione del catetere se la positività dei flaconi con sangue prelevato da $\mathrm{CVC}$ viene rilevata almeno 120 minuti prima rispetto alla positività dei flaconi con sangue prelevato da vena periferica $(5,11)$.

\section{ESAME MICROBIOLOGICO DELLA PUNTA DEL CATETERE VENOSO CENTRALE:}

sono state studiate 217 punte di CVC nell'arco di 8 anni, dal 1996 al 2004. I cateteri sono stati rimossi per sospetta infezione associata oppure per fine utilizzo. Sono state utilizzate le seguenti metodiche:

- Esame colturale quantitativo secondo Cleri;

- Esame colturale qualitativo (coltura per 7 giorni in Tripticase Soy Broth).

Esame colturale quantitativo secondo Cleri (CLERI):

E stata utilizzata la Tecnica di Cleri come ripresa dall'Autore (6) dal Gruppo AMCLI di Studio sulla Diagnostica delle Infezioni da Catetere Vascolare (12).

Il catetere è stato rimosso dal paziente in condizioni di asepsi. Con pinza e forbici sterili è stata tagliata la punta del catetere $(5 \mathrm{~cm})$ che viene inserita in apposito contenitore sterile (provetta con $5 \mathrm{ml}$ di terreno liquido di trasporto (Tripticase Soy Broth: TSB) e inviata rapidamente al Servizio di Microbiologia oppure conservata nella provetta con il terreno liquido di trasporto in frigorifero a $+4^{\circ} \mathrm{C}$ ed inviata entro 18 ore al Servizio di Microbiologia.

In Laboratorio si è proceduto come segue:

- agitazione su vortex della provetta contenente la punta del catetere per 1 minuto;

- semina su 3 diverse piastre di agar sangue (Columbia agar $+5 \%$ di sangue di montone) rispettivamente di $1 \mu 1,10 \mu 1$ e $100 \mu 1$ di terreno liquido di trasporto;
- incubazione delle piastre a $37^{\circ} \mathrm{C}$ in atmosfera al $5 \%$ di $\mathrm{CO}_{2}$ per 18 ore, prolungando l'incubazione fino a 48 ore in caso di negatività;

- conta del numero di colonie cresciute e moltiplicazione per i fattori di diluizione (per le 3 diluizioni rispettivamente $\mathrm{x} 1000 \times 5, \times 100 \times 5$, $\mathrm{x} 10 \mathrm{x} 5)$. Il valore soglia significativo per infezione della cannula è $\geq 1000 \mathrm{CFU} / \mathrm{ml}$.

Esame colturale qualitativo (TSB):

Dopo aver eseguito la metodica di Cleri, il campione in TSB viene posto in incubazione a $37^{\circ} \mathrm{C}$ e tenuto in osservazione per 7 giorni:

- Se il brodo si presentava torbido dopo il primo giorno o nei giorni successivi, sono state eseguite sottocolture su terreni solidi.

- Se dopo le prime 24 ore la brodocoltura si presenta limpida, veniva comunque eseguita una sottocoltura di controllo su Agar Sangue. Allo scadere del settimo giorno, se il TSB si manteneva limpido, veniva comunque eseguita un'ultima coltura di controllo su Agar Sangue.

\section{TECNICHE BATTERIOLOGICHE E IDENTIFICAZIONE DEI MICRORGANISMI:}

sono state utilizzate le tecniche batteriologiche di base. I microrganismi isolati sono stati identificati con il Sistema automatico ATB (bioMérieux) e con il Sistema Manuale API (bioMérieux). Per le identificazioni sono stati eseguiti Controlli di Qualità Interni utilizzando i seguenti Ceppi:

Staphylococcus aureus ATCC 29213,

Enterococcus faecalis ATCC 29212,

Escherichia coli ATCC 25922,

Pseudomonas aeruginosa ATCC 27853.

Durante il periodo dello studio sono stati eseguiti Controlli di Qualità Esterni (BIODEV e UKNEQAS).

\section{RISULTATI}

A. Raccolta e riesame dei dati della nostra casistica relativi alla diagnostica microbiologica delle infezioni CVC associate:

In tabella 1 sono riportati in sintesi i risultati ottenuti con l'esame colturale quantitativo del CVC secondo Cleri (CLERI), con l'esame colturale qualitativo della punta del CVC (TSB) e con l'emocoltura qualitativa. I risultati sono suddivisi in 4 gruppi determinati dalla carica microbica dell'esame quantitativo del CVC. Inoltre, di ogni gruppo, vengono evidenziati i casi con emocolture positive per lo stesso microrganismo isolato nell'esame della punta CVC e i casi con emocolture con esito negativo. 
Tabella 1. risultati ottenuti con l'esame colturale quantitativo (CLERI) e qualitativo (TSB) della punta del CVC e con l'emocoltura qualitativa suddivisi in 4 gruppi determinati dalla carica microbica dell'esame quantitativo del CVC (in ogni gruppo, vengono evidenziati i casi con emocolture positive per lo stesso microrganismo isolato nell'esame della punta CVC e i casi con emocolture con esito negativo):

Gruppo 1: punte CVC positive con la metodica di Cleri con carica $\geq 1000 \mathrm{cfu} / \mathrm{mL}$ e positive con l'esame qualitativo (TSB);

Gruppo 2: punte CVC positive con la metodica di Cleri con carica $<1000 \mathrm{cfu} / \mathrm{mL}$ e positive con l'esame qualitativo (TSB);

Gruppo 3: punte CVC negative con la metodica di Cleri e positive con l'esame qualitativo (TSB);

Gruppo 4: punte CVC negative con la metodica di Cleri e negative con la metodica qualitativa (TSB).

Tabella I

\begin{tabular}{lcccccc}
\hline & CLERI & TSB & N CVC & EMOCOLTURE \\
\hline & ESITO & ESITO & $\begin{array}{c}\text { Totale } \\
217\end{array}$ & ESITO & N & $\%$ \\
& & & & & \\
\hline \multirow{2}{*}{ Gruppo I } & $\geq 1000$ & \multirow{2}{*}{ Pos. } & \multirow{2}{*}{66} & Pos. & 47 & 71.2 \\
& cfu/mL & & & Neg. & 19 & 28,8 \\
\hline \multirow{2}{*}{ Gruppo 2 } & $<1000$ & \multirow{2}{*}{ Pos. } & \multirow{2}{*}{4} & Pos. & I & 25 \\
& cfu/mL & & & Neg. & 3 & 75 \\
\hline \multirow{2}{*}{ Gruppo 3 } & \multirow{2}{*}{ Neg. } & \multirow{2}{*}{ Pos. } & \multirow{2}{*}{12} & Pos. & 4 & 33.3 \\
& & & Neg. & 8 & 66,7 \\
\hline Gruppo 4 & Neg. & Neg. & 135 & & & \\
\hline
\end{tabular}

Nelle tabelle 2a e 2b si riporta l'epidemiologia dei germi isolati da punta di CVC con carica $\geq 1000$ $\mathrm{cfu} / \mathrm{mL}$ dell'esame quantitativo e con esame qualitativo della punta positivo (tabella 2 a casi con emocoltura positiva per lo stesso microrganismo isolato nell'esame della punta CVC e Tabella $2 \mathrm{~b}$ casi con emocoltura negativa).

Tabella 2a. Germi isolati da punta di CVC con carica $\geq 1000$ cfu/mL dell'esame quantitativo e con esame qualitativo della punta positivo, con emocoltura positiva per lo stesso microrganismo isolato nell'esame della punta CVC.

\begin{tabular}{ll}
\multicolumn{2}{c}{$\begin{array}{c}\text { Epidemiologia germi isolati su } \\
\text { Cleri carica } \geq \mathbf{1 0 0 0} \text { - TSB pos. - EMO pos. }\end{array}$} \\
\hline GERMI ISOLATI & $\mathbf{N}$ \\
\hline Staphylococcus epidermidis & 20 \\
\hline Staphylococcus aureus & 13 \\
\hline Pseudomonas aeruginosa & 3 \\
\hline Enterococcus faecalis & 2 \\
\hline Escherichia coli & $\mathrm{I}$ \\
\hline Morganella morganii & $\mathrm{I}$ \\
\hline Acinetobacter spp & $\mathrm{I}$ \\
\hline Acinetobacter johnsonii & $\mathrm{I}$ \\
\hline Candida albicans & 5 \\
\hline Candida parapsilosis & 5 \\
\hline Candida guilliermondii & $\mathrm{I}$
\end{tabular}

Tabella 2b. Germi isolati da punta di CVC con carica $\geq 1000$ cfu/mL dell'esame quantitativo e con esame qualitativo della punta positivo con emocoltura negativa.

\begin{tabular}{|c|c|}
\hline \multicolumn{2}{|c|}{$\begin{array}{l}\text { Epidemiologia germi isolati su I } 9 \text { campioni } \\
\text { Cleri carica } \geq 1000 \text { - TSB pos. - EMO neg. }\end{array}$} \\
\hline GERMI ISOLATI & $\mathbf{N}$ \\
\hline taphylococcus epidermidis & 18 \\
\hline taphylococcus aureus & $\mathrm{I}$ \\
\hline taphylococcus haemolyticus & 2 \\
\hline
\end{tabular}

In riferimento ai germi isolati da punta di CVC con carica $<1000 \mathrm{cfu} / \mathrm{mL}$ dell'esame quantitativo e con esame qualitativo della punta positivo (con emocoltura positiva per lo stesso microrganismo isolato nell'esame della punta $\mathrm{CVC}$ ), l'unico isolamento è di $1 S$. epidermidis. In riferimento ai germi isolati da punta di CVC con carica $<1000$ $\mathrm{cfu} / \mathrm{mL}$ dell'esame quantitativo e con esame qualitativo della punta positivo con emocoltura negativa abbiamo 3 casi di isolamento di $S$. epidermidis.

Nelle tabelle 3a e 3b si riporta l'epidemiologia dei germi isolati da punta CVC con esame quantitativo negativo e con esame qualitativo della punta positivo (tabella 3 a casi con emocoltura positiva per lo stesso microrganismo isolato nell'esame della punta $\mathrm{CVC}$ e tabella $3 \mathrm{~b}$ casi con emocoltura negativa).

Tabella 3a. Germi isolati da punta CVC con esame quantitativo negativo e con esame qualitativo della punta positivo con emocoltura positiva per lo stesso microrganismo isolato nell'esame della punta CVC.

\begin{tabular}{ll}
\multicolumn{2}{c}{$\begin{array}{c}\text { Epidemiologia germi isolati su } 4 \text { campioni } \\
\text { CLERI neg. - TSB pos. - EMO Pos. }\end{array}$} \\
\hline GERMI ISOLATI & $\mathbf{N}$ \\
\hline Staphylococcus epidermidis & $\mathrm{I}$ \\
\hline Kocuria kristinae & $\mathrm{I}$ \\
\hline Escherichia coli & $\mathrm{I}$ \\
\hline Acinetobacter baumanii & $\mathrm{I}$ \\
\hline
\end{tabular}

Tabella 3b. Germi isolati da punta CVC con esame quantitativo negativo e con esame qualitativo della punta positivo casi con emocoltura negativa.

Epidemiologia germi isolati su 8 campioni CLERI neg. - TSB pos. - EMO neg.

\begin{tabular}{ll}
\hline GERMI ISOLATI & N \\
\hline Staphylococcus epidermidis & 5 \\
\hline Cocchi gram positivi non id. & 2 \\
\hline Corynebacterium spp & I \\
\hline Candida parapsilosis & I \\
\hline
\end{tabular}

In tabella 4 e in tabella 5 si riportano i risultati di uno studio condotto nel nostro Laboratorio (9) con il quale si è voluta approfondire la patogenicità dello $S$. epidermidis (il microrganismo più frequentemente isolato nelle infezioni associate al 
cateterismo vascolare) per confrontare questi risultati con alcuni risultati ottenuti con l'esame colturale quantitativo del CVC secondo Cleri (CLERI), con l'esame colturale qualitativo della punta del CVC (TSB) e con l'emocoltura qualitativa. In questo studio sono state utilizzate le seguenti metodiche per la valutazione di alcuni fattori di virulenza:

a- per la valutazione quantitativa della produzione di biofilm (con un metodo quantitativo spettrofotometrico su piastre da microtitolazione (Christensen modificato);

b- per studiarne l'abilità di mediare l'emoagglutinazione (associata con l'abilità di aderire ai biomateriali);

c- per la valutazione qualitativa della formazione del biofilm (su agar Rosso Congo).

Sono stati studiati 44 ceppi di $S$. epidermidis isolati da punte di catetere venoso centrale $(n=24)$, da emocolture $(n=10)$, da urinocolture $(n=5)$ e da tamponi cutanei $(n=5)$. Il 29.5\% degli S. epidermidis isolati risultano essere forti produttori di biofilm, il $31.8 \%$ presenta un elevato titolo di emoagglutinazione $(\geq 1 / 16)$ e il $18.1 \%$ forma su agar Rosso Congo colonie nere dall'aspetto secco tipiche dei ceppi produttori di biofilm (tabella 4). Gli isolati batterici che sono forti produttori di biofilm con il metodo quantitativo, risultano avere, nella maggior parte dei casi, elevati titoli di emoagglutinazione. I risultati mettono in evidenza che la maggior parte dei ceppi di S. epidermidis forti produttori di biofilm e con un elevato titolo di emoagglutinazione sono isolati da punte di catetere venoso centrale (10).

Tabella 4. Risultati dello studio con test fenotipici di 44 ceppi di S. epidermidis.

\begin{tabular}{|c|c|c|c|}
\hline \multicolumn{4}{|c|}{$\begin{array}{l}\text { RISULTATI DEI TRE TEST FENOTIPICI SUI CEPPI } \\
\text { DI S. epidermidis }\end{array}$} \\
\hline & $\begin{array}{c}\text { FORTI } \\
\text { produttori }\end{array}$ & $\begin{array}{l}\text { DEBOLI } \\
\text { produttori }\end{array}$ & $\begin{array}{c}\text { NON } \\
\text { produttori }\end{array}$ \\
\hline \multirow[t]{2}{*}{$\begin{array}{l}\text { PRODUZIONE DI BIOFILM } \\
\text { (metodo quantitativo) }\end{array}$} & $29.5 \%$ & $50 \%$ & $20.5 \%$ \\
\hline & $\begin{array}{l}\text { ELEVATO Titolo } \\
>>1 / 16\end{array}$ & $\begin{array}{l}\text { BASSO Titolo } \\
\quad<1 / 8\end{array}$ & NEGATIVO \\
\hline \multirow[t]{2}{*}{ EMOAGGLUTINAZIONE } & $31.8 \%$ & $18.2 \%$ & $50 \%$ \\
\hline & $\begin{array}{l}\text { CEPPI POSITIVI } \\
\text { (colonie NERE) }\end{array}$ & $\begin{array}{l}\text { CEPPI NEGATIVI } \\
\text { (colonie ROSSE) }\end{array}$ & \\
\hline \multicolumn{4}{|c|}{ MORFOLOGIA DELLE COLONIE } \\
\hline SU CONGO RED AGAR (CRA & $18.1 \%$ & $81.9 \%$ & \\
\hline
\end{tabular}

Con le metodiche sopra descritte sono stati studiati 4 ceppi di $S$. epidermidis isolati da punte di catetere venoso centrale risultate negative con la tecnica quantitativa di Cleri, ma positive con l'esame qualitativo, per valutare se le caratteristiche di adesività e di produzione di biofilm potevano essere correlate con la discrepanza di risultato tra la metodica quantitativa di Cleri e l'esame qualitativo (tabella 5)(10).

Tabella 5. Risultati dello studiati con test fenotipici di 4 ceppi di S. epidermidis isolati da punte di catetere venoso centrale risultate negative con la tecnica quantitativa di Cleri, ma positive con l'esame qualitativo.

\begin{tabular}{lccc}
\hline \multicolumn{4}{c}{ RISULTATI TEST FENOTIPICI } \\
\hline CEPPO & ROSSO CONGO & EMOAGGLUTINAZ. & BIOFILM \\
\hline SII5 & Positivo & I / I28 & +++ \\
\hline S166 & Negativo & Negativo & Negativo \\
\hline SI9I & Positivo & I / I28 & +++ \\
\hline S248 & Negativo & Negativo & Negativo \\
\hline
\end{tabular}

Riportiamo a questo punto, perché ci sembrano un approfondimento interessante, i risultati di un altro studio $(2,7)$, condotto dal nostro Laboratorio in collaborazione con l'Istituto di Microbiologia dell'Università di Trieste, in cui abbiamo analizzato gli S. epidermidis (il microrganismo più frequentemente isolato nelle infezioni associate al cateterismo vascolare) isolati dalla punta del CVC e dal sangue nello stesso paziente (in otto pazienti in totale) per verificare se derivavano dallo stesso ceppo. Le punte dei CVC sono state sottoposte ad esame quantitativo (utilizzando la metodica di CLERI) e ad esame qualitativo (mediante coltura in terreno TSB) con le modalità già viste, il sangue è stato valutato con emocoltura quantitativa. Le tecniche di biologia molecolare utilizzate sono state PCR-ribotyping, ribotyping e Pulse-field gel electrophoresis (PFGE). PCR-ribotyping non è stata in grado di distinguere i diversi ceppi, mentre il ribotyping e la PFGE hanno dimostrato la corrispondenza fra i ceppi isolati dalla punta e dal sangue dello stesso paziente $(2,7)$.

\section{B- Allestimento ed esecuzione dell'emocoltura quantitativa e dell'emocoltura basata sul "dif- ferential time to positivity"}

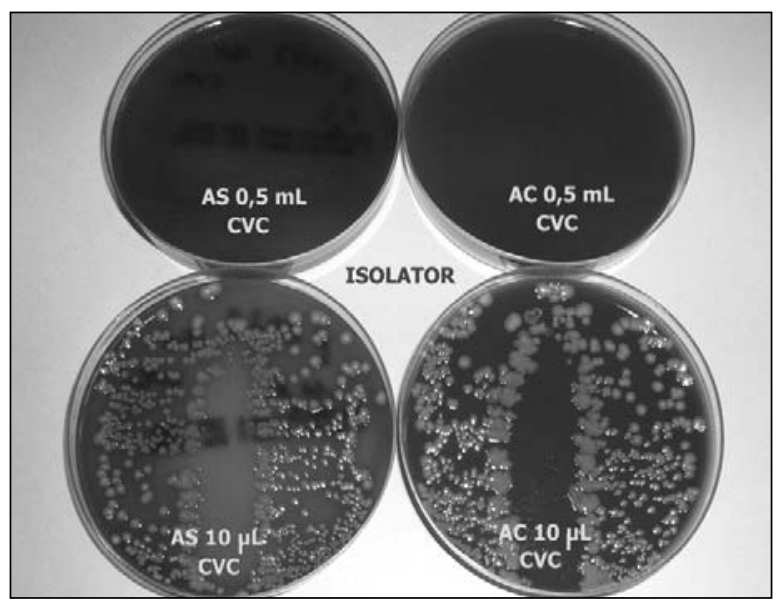

Figura I. Immagini della crescita sulle piastre relative al $3^{\circ}$ caso. 
Sono stati studiati, utilizzando entrambe le metodiche, 20 casi. Di questi, 3 hanno dato esito positivo. Nella figura I si riportano le immagini della crescita sulle piastre relative al $3^{\circ}$ caso. In tabella 6 sono riassunti i risultati mettendo a confronto entrambe le metodiche. frequentemente isolato nelle infezioni associate al cateterismo vascolare) e presente in alcuni di questi casi di discrepanza, non sembrerebbe avvallare questa ipotesi (tabella 4 e tabella 5);

- Il numero di CVC infetti (carica $>1000 \mathrm{cfu} / \mathrm{ml}$ secondo la Tecnica di Cleri) risulta elevato (66 su

Tabella 6. Risultati dell'emocoltura quantitativa e dell'emocoltura basata sul "differential time to positivity" relativi ai casi positivi.

\begin{tabular}{|c|c|c|c|c|c|}
\hline & PRELIEVO & $\begin{array}{c}\text { EMOCOLTURA } \\
\text { DTP }\end{array}$ & $\begin{array}{l}\text { TEMPO DI } \\
\text { POSITIVITA' }\end{array}$ & $\begin{array}{c}\text { ISOLATOR } \\
0.5 \mathrm{~mL}\end{array}$ & $\begin{array}{c}\text { ISOLATOR } \\
\mu 10 \mathrm{~mL}\end{array}$ \\
\hline $1^{\circ} \mathrm{CASO}$ & $\begin{array}{l}\text { Periferico } \\
\text { CVC }\end{array}$ & $\begin{array}{l}\text { S. sciuri } \\
\text { S. sciuri }\end{array}$ & $\begin{array}{l}20 \text { ore } \\
7 \text { ore }\end{array}$ & $\begin{array}{l}\text { Neg. } \\
\text { S. sciuri }\end{array}$ & S. sciuri \\
\hline $2^{\circ} \mathrm{CASO}$ & $\begin{array}{l}\text { Periferico } \\
\text { CVC }\end{array}$ & $\begin{array}{c}\text { Neg. } \\
\text { E. cloacae }\end{array}$ & 22 ore & $\begin{array}{l}\text { Neg. } \\
\text { Neg. }\end{array}$ & E. cloaca................ \\
\hline $3^{\circ} \mathrm{CASO}$ & $\begin{array}{l}\text { Periferico } \\
\text { CVC }\end{array}$ & $\begin{array}{c}\text { Neg. } \\
\text { S. hominis }\end{array}$ & 50 ore & $\begin{array}{c}\text { Neg. } \\
\text { S. hominis }\end{array}$ & Neg. \\
\hline
\end{tabular}

\section{DISCUSSIONE}

A-Raccolta e riesame dei dati della nostra casistica relativi alla diagnostica microbiologica delle infezioni CVC associate:

- Validità e praticità della Tecnica di Cleri: in caso di risultato significativo con la Tecnica di Cleri le emocolture sono positive nel $71.2 \%$; tali percentuali scendono al $25 \%$ e al $33.3 \%$ in caso di risultato non significativo o negativo con la stessa Tecnica (tabella 1). Tali percentuali forse potrebbero essere ulteriormente migliorate con una ulteriore maggiore attenzione alla fase preanalitica (esatta lunghezza del CVC, corretta modalità di conservazione e tempistica di invio; correttezza nei tempi e modi di prelievo delle emocolture). Al contrario, dai nostri dati, la Tecnica di coltura in terreno liquido ha percentuali di falsi positivi che vanno dal $66.7 \%$ al 75\% (tabella 1). Nello studio riportato $(2,7)$, condotto dal nostro Laboratorio in collaborazione con l'Istituto di Microbiologia dell'Università di Trieste, è stata dimostrata l'identità dei ceppi di $S$. epidermidis isolati dalla punta del CVC e dalle emocolture;

- Alla luce dei casi, anche se pochi, con risultato non significativo o negativo con la tecnica di Cleri, ma positivi con la tecnica di coltura in terreno liquido e con emocolture positive (tabella 1 e tabella $3 a$ ), ci sentiamo però di tenere abbinate le due tecniche (data anche la loro semplicità), tanto più in pazienti immunocompromessi nei quali non diagnosticare un'infezione potrebbe assumere un significato particolarmente grave. La Tecnica di Cleri rimane comunque un riferimento interpretativo. Abbiamo voluto approfondire questa discrepanza tra le due tecniche verificando se potesse essere correlata alla capacità di adesione dei diversi ceppi. I risultati dello studio riportato condotto nel nostro Laboratorio (10), con il quale si è voluta approfondire la patogenicità dello $S$. epidermidis (il microrganismo più
217 CVC analizzati con una percentuale del $30.4 \%$ )(tabella 1) rispetto alle percentuali attese in base ai dati della letteratura (CVC infetti pari a 3-5\%). Tale dato rientra invece pienamente nei valori attesi se rapportato non ai CVC analizzati (che nella gran parte dei casi vengono inviati al Servizio di Microbiologia perché sospetti infetti), ma al numero di cateteri impiantati (tenendo conto che la gran parte dei CVC sospetti infetti viene inviata al Servizio di Microbiologia)(66 su 4496 CVC impiantati nel periodo 1996-2004 pari a una percentuale dell' $1.5 \%$ );

- Epidemiologia dei microrganismi isolati: gli Stafilococchi risultano essere i microrganismi più frequentemente isolati, in particolare $S$. epidermidis, seguito da $S$. aureus. Sono però presenti anche altri potenziali patogeni come $P$. aeruginosa, E. faecalis, E. coli, M. morganii, Acinetobacter spp, Candida spp. Possono essere coinvolti anche microrganismi di raro riscontro come Kocuria kristinae (tabella 2a e 3a)(3). Siccome lo $S$. epidermidis risulta essere anche il più frequente tra i contaminanti (tabella $2 \mathrm{~b}$ e $3 \mathrm{~b}$ ), l'utilizzo di una tecnica quantitativa come la Tecnica di Cleri risulta di grande utilità nel discriminare $\mathrm{i}$ contaminanti dai microrganismi realmente causa di infezione del catetere.

B-Allestimento ed esecuzione dell'emocoltura quantitativa e dell'emocoltura basata sul "differential time to positivity":

Nella nostra esperienza, pur iniziale e numericamente contenuta, le due metodiche sono risultate di esecuzione abbastanza semplice. Tuttavia per entrambe ci sembra molto importante rispettare il protocollo di esecuzione sia nella fase pre-analitica che nella fase analitica. Per esempio, nella nostra esperienza, con riferimento all'emocoltura quantitativa, è stato fondamentale utilizzare nella valutazione del sangue prelevato da CVC la semina in due diversi terreni (AS e AC), utilizzando due diverse quantità di sangue. Nel $2^{\circ}$ caso (tabella 6 ) 
infatti, si può notare che non vi è crescita nella piastra inoculata con $0,5 \mathrm{~mL}$ di sangue, mentre la crescita è rilevabile nella piastra inoculata con $10 \mu \mathrm{L}$ di sangue (verosimilmente nella prima per la maggior quantità di sangue, vi è anche una maggior quantità di antibiotico che il paziente stava assumendo per esigenze cliniche, che inibisce la crescita del microrganismo) (Foto 1 ). Nel $3^{\circ}$ caso (tabella 6) si nota al contrario crescita solo nella piastra inoculata con $0,5 \mathrm{~mL}$ di sangue, verosimilmente per una bassa carica del microrganismo (nella piastra sono state contate solo 30 colonie). Nei casi descritti l'uso combinato di entrambe le piastre con le due quantità di sangue ha permesso di evidenziare le positività che altrimenti sarebbero sfuggite.

\section{CONCLUSIONI}

A- Raccolta e riesame dei dati della nostra casistica relativi alla diagnostica microbiologica delle infezioni CVC associate:

- la tecnica di Cleri è una tecnica molto valida e pratica;

- tenere abbinate le due tecniche quantitativa e qualitativa consente di aumentare ulteriormente la sensibilità, già buona, della sola tecnica di Cleri;

- gli Stafilococchi risultano essere i microrganismi più frequentemente isolati, in particolare Staphylococcus epidermidis. Possono però essere coinvolti anche altri potenziali patogeni (come Pseudomonas aeruginosa, Enterococcus faecalis, Candida spp ed enterobatteri) e anche microrganismi di raro riscontro (come Kocuria kristinae).

B- $\underline{\text { Allestimento ed esecuzione dell'emocoltura }}$ quantitativa e dell'emocoltura basata sul "differential time to positivity":

- tecniche, nella nostra esperienza e nella nostra casistica, di esecuzione abbastanza semplice e con una buona corrispondenza di risultato tra loro;

- per entrambe ci sembra molto importante rispettare il protocollo di esecuzione sia nella fase pre-analitica che nella fase analitica;

- tenendo presenti pregi e limiti dell'una e dell'altra metodica entrambe sono molto valide e utili per la diagnostica microbiologica delle infezioni CVC associate; 1'utilizzo contemporaneo di entrambe le metodiche, se possibile, risulta utile e più completo.

\section{BIBLIOGRAFIA}

1. Basaglia G, Stocco S, Sperandio P, Pancino A, Ligato E, De Paoli P. Infezioni associate al cateterismo vascolare in soggetti immunocompromessi: studio epidemiologico e diagnostico. XXVI Congresso Nazionale Associazione Microbiologi Clinici Italiani (AMCLI), 4-7/11/1997, Napoli. Microbiologia Medica 1998; 13 (2): 471-2.

2. Basaglia G, Moras L, Lagatolla C, Tonin E, De Paoli P.
Molecular epidemiology of Staphylococcus epidermidis isolated from central venous cathether infections. 101 General Meeting, American Society of Microbiology, 20-24/05/2001, Orlando.

3. Basaglia G, Carretto E, Barbarini D, Moras L, Scalone S, Marone P, De Paoli P. Catheter-related bacteremia due to Kokuria kristinae in a patient with ovarian cancer. J Clin Microbiol 2002; 40 (1): 311-3.

4. Basaglia G, Stocco Calzavara S, Pancino A, Sperandio P, Tomasini ML, De Paoli P. Diagnostica microbiologica delle infezioni associate ai CVC: studio in pazienti immunocompromessi. XXXIV Congresso Nazionale Associazione Microbiologi Clinici Italiani (AMCLI), 11-14/10/2005, Roma. Microbiologia Medica 2005; 20 (3): 170.

5. Blot F, Nitenberg G, Chachaty E, Raynard B, Germann N, Antoun S, Laplanche A, Brun-Buisson C, Tancrede C. Diagnosis of catheter-related bacteraemia: a prospective comparison of the time to positivity of hub-blood versus peripheral blood cultures. Lancet 1999; 354: 1071-7.

6. Cleri DJ, Corrado ML, Seligman SJ. Quantitative culture of intravenous catheters and other intravascular inserts. J Infect Dis 1980; 141(6): 781-6.

7. De Paoli P, Basaglia G, Moras L. Infezioni associate al cateterismo vascolare in soggetti immunocompromessi. $20^{\circ}$ Congresso Nazionale della Società Italiana di Chemioterapia (SIC), 28/11-1/12/1999, Torino.

8. Kluger DM, Maki DG. The relative risk of intravascular device related blood-stream infections in adults [Abstract]. In: Abstracts of the $39^{\text {th }}$ Interscience Conference on Antimicrobial Agents and Chemotherapy, San Francisco, CA, 26-29 September 1999. Abstract no. 514.

9. Lagatolla C, De Paoli P, Basaglia GC, Moras L, Tonin E, Dolzani L, Lavenia A, Monti Bragadin C. Tipizzazione molecolare di ceppi di Staphylococcus epidermidis isolati da batteriemie catetere-correlate. $28^{\circ}$ Congresso Nazionale della Società Italiana di Microbiologia (SIM), 19-22/10/2000, Jesi (AN).

10. Moras L, Basaglia G, Pancino A, Stocco S, De Paoli P. Caratterizzazione fenotipica di $S$. epidermidis isolati da CVC in pazienti immunocompromessi. XXXI Congresso Nazionale Associazione Microbiologi Clinici Italiani (AMCLI), 17-20/09/2002, Rimini. Microbiologia Medica 2002; 17 (2): 141.

11. Raad I, Hanna H, Alakech B, Chatzinikolaou I, Johnson MM, Tarrand J. Differential time to positivity: a useful method for diagnosing catheter-related bloodstream infections. Ann Intern Med 2004; 140: 18-25.

12. Rescaldani R, Giltri G, Bramati S, Farina C, Goglio A, Grigis A, Marone P, Monzillo V e Gruppo di Studio sulla Diagnostica delle Infezioni da Catetere Vascolare (GSDICV). Infezioni associate al cateterismo vascolare: consensus sull'iter diagnostico microbiologico. Microbiologia Medica 1997; 12 (1): XIV-XXII.

\section{Basaglia Giancarlo}

Struttura Complessa di Microbiologia, Immunologia e Virologia

Centro di Riferimento Oncologico, Istituto Nazionale Tumori - IRCCS Via Pedemontana Occidentale, 12 33081 Aviano (Pordenone)

Tel 0434/659406 (Studio) - 0434/659421

(Segreteria) - Fax: 0434/659402

E-mail: gbasaglia@cro.it 